\title{
Embelin's Versatile Photochemistry \\ Makes it a Potent Photosensitizer for Photodynamic Therapy
}

\author{
Michael Rogo Opata and Andreas Dreuw* \\ Interdisciplinary Center for Scientific Computing, Heidelberg University, Im \\ Neuenheimer Feld 205, D-69120 Heidelberg, Germany. \\ E-mail:dreuw@uni-heidelberg.de
}

SUPPLEMENTARY INFORMATION 


\section{Cartesian Coordinates of embelin model}

$\begin{array}{lrrr}\mathrm{O} & 2.49547600 & 0.68367400 & -0.00002100 \\ \mathrm{O} & -2.18465900 & 1.22056800 & 0.00002000 \\ \mathrm{O} & -2.56847800 & -1.32262900 & -0.00002500 \\ \mathrm{O} & 2.11248600 & -1.85607800 & 0.00001800 \\ \mathrm{C} & 0.31782600 & 2.65263000 & -0.00000100 \\ \mathrm{C} & 0.18125700 & 1.16052600 & -0.00000500 \\ \mathrm{C} & 1.22407300 & 0.29758400 & -0.00001900 \\ \mathrm{C} & -1.14087700 & 0.57493300 & 0.00002200 \\ \mathrm{C} & -1.30287200 & -0.93471500 & 0.00000700 \\ \mathrm{C} & 1.06931900 & -1.21050800 & 0.00000600 \\ \mathrm{C} & -0.25218300 & -1.77826800 & 0.00000900 \\ \mathrm{H} & 1.36389400 & 2.94672000 & -0.00005000 \\ \mathrm{H} & -0.16997700 & 3.08506500 & -0.87501600 \\ \mathrm{H} & -0.36365300 & -2.85213500 & 0.00000900 \\ \mathrm{H} & 3.01241600 & -0.15075200 & -0.00000700 \\ \mathrm{H} & -3.09067400 & -0.49131800 & -0.00009000 \\ \mathrm{H} & -0.16985400 & 3.08505800 & 0.87509600\end{array}$

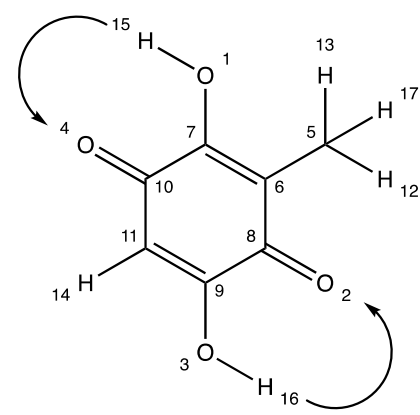

Figure S1:Truncated model of embelin used in calculations. The H15 and H16 were simultaneously moved towards 04 and 02 respectively in the dynamic photochemistry studies. 
Static optical properties and vertical excited states
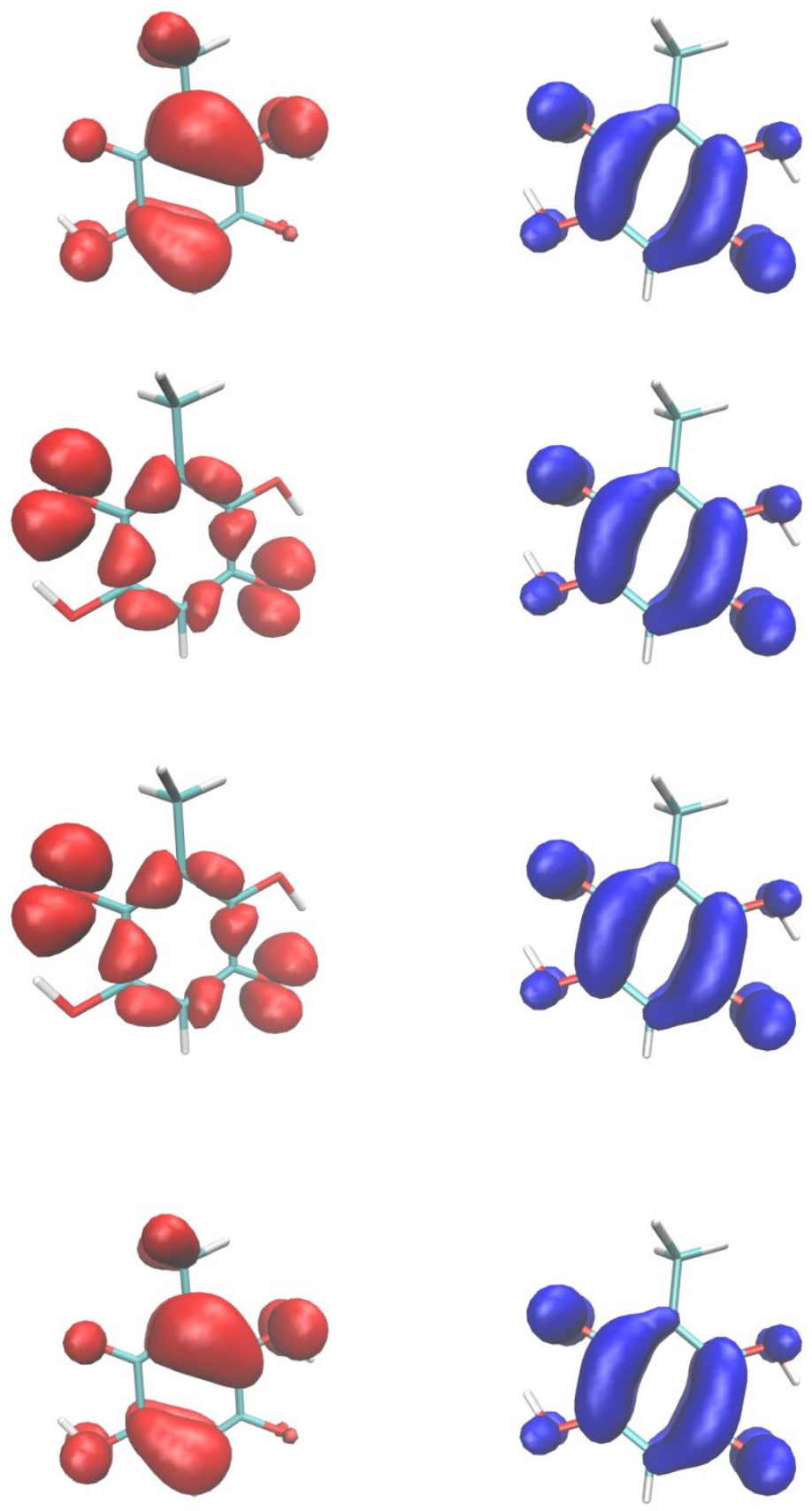

Figure S2: Detachment (red) and attachment (blue) densities of the T1 $\left(\pi \pi^{*}\right), T 2\left(n \pi^{*}\right), T 3\left(n \pi^{*}\right)$ and $T 4\left(\pi \pi^{*}\right)$ (top to bottom) transitions of embelin at the level of TDDFT/CAM-B3LYP 


\begin{tabular}{|l|c|c|c|c|c|c|}
\hline State & ADC(2) & ADC(3) & BLYP & B3LYP & CAM-B3LYP & BHHLYP \\
\hline $\mathbf{S}_{\mathbf{1}}$ & $3.03(0.00)$ & $3.09(0.00)$ & $2.32(0.00)$ & $2.86(0.00)$ & $3.08(0.00)$ & $3.3567(0.00)$ \\
\hline $\mathbf{S}_{\mathbf{2}}$ & $3.22(0.00)$ & $3.63(0.00)$ & $2.70(0.00)$ & $3.24(0.00)$ & $3.66(0.00)$ & $3.8784(0.00)$ \\
\hline $\mathbf{S}_{\mathbf{3}}$ & $3.46(0.00)$ & $3.90(0.00)$ & $3.00(0.00)$ & $3.55(0.00)$ & $3.97(0.00)$ & $4.209(0.00)$ \\
\hline $\mathbf{S}_{\mathbf{4}}$ & $4.57(0.39)$ & $4.90(0.46)$ & $3.74(0.13)$ & $4.57(0.39)$ & $4.81(0.33)$ & $5.13(0.388)$ \\
\hline $\mathbf{T}_{\mathbf{1}}$ & 1.77 & 2.29 & 1.68 & 1.87 & 1.99 & 2.01 \\
\hline $\mathbf{T}_{\mathbf{2}}$ & 2.26 & 2.77 & 2.33 & 2.49 & 2.50 & 2.34 \\
\hline $\mathbf{T}_{\mathbf{3}}$ & 3.36 & 3.35 & 2.41 & 2.72 & 3.12 & 3.31 \\
\hline $\mathbf{T}_{\mathbf{4}}$ & 3.50 & 3.64 & 2.61 & 2.77 & 3.47 & 3.64 \\
\hline
\end{tabular}

Table S1: Complete Gas phase vertical excitation energies ( $\mathrm{eV}$ ) of the four lowest singlet and triplet states of embelin at the level of $A D C(2), A D C(3)$ and TDDFT with the BLYP, B3LYP, CAM-B3LYPand BHHLYPXC-functionals. Oscillator strengths are given in parenthesis. 


\section{Exciton properties of the $S_{1}$ state}

\begin{tabular}{|l|l|c|c|c|c|}
\hline S1 & Method & $\mathbf{E}$ & $\mathbf{P R}_{\text {NTO }}$ & $\mathbf{r}_{\mathrm{e}-\mathrm{h}}$ & $\mathbf{R}$ \\
\hline \multirow{4}{*}{} & ADC(2) & 3.03 & 1.0252 & 0.29 & 0.033 \\
\cline { 2 - 6 } & ADC(3) & 3.09 & 1.0296 & 0.22 & 0.037 \\
\cline { 2 - 6 } & B3LYP & 2.72 & 1.0363 & 0.35 & -0.027 \\
\cline { 2 - 6 } & CAM-B3LYP & 3.08 & 1.0355 & 0.35 & -0.027 \\
\hline
\end{tabular}

\begin{tabular}{|l|l|c|c|c|c|}
\hline S2 & Method & $\mathbf{E}$ & $\mathbf{P R}_{\text {NTO }}$ & $\mathbf{r}_{\mathrm{e}-\mathrm{h}}$ & $\mathbf{R}$ \\
\hline \multirow{8}{*}{} & ADC(2) & 3.22 & 1.08 & 0.41 & 0.189 \\
\cline { 2 - 6 } & ADC(3) & 3.63 & 1.18 & 0.08 & 0.28 \\
\cline { 2 - 6 } & B3LYP & 3.22 & 1.023 & 0.30 & 0.10 \\
\cline { 2 - 6 } & CAM-B3LYP & 3.26 & 1.03 & 0.29 & -0.03 \\
\hline
\end{tabular}

\begin{tabular}{|l|l|c|c|c|c|}
\hline S3 & Method & $\mathbf{E}$ & PR $_{\text {NTO }}$ & $\mathbf{r}_{\mathrm{e}-\mathrm{h}}$ & $\mathbf{R}$ \\
\hline \multirow{4}{*}{} & ADC(2) & 3.50 & 1.1 & 0.34 & 0.22 \\
\cline { 2 - 6 } & ADC(3) & 3.89 & 1.23 & 0.05 & 0.32 \\
\cline { 2 - 6 } & B3LYP & 3.54 & 1.04 & 0.23 & 0.10 \\
\cline { 2 - 6 } & CAM-B3LYP & 3.60 & 1.04 & 0.21 & 0.12 \\
\hline
\end{tabular}

\begin{tabular}{|l|l|c|c|c|c|}
\hline S4 & Method & $\mathbf{E}$ & PR $_{\text {NTO }}$ & $\mathbf{r}_{\mathrm{e}-\mathrm{h}}$ & $\mathbf{R}$ \\
\hline \multirow{4}{*}{} & ADC(2) & 4.57 & 1.09 & 0.19 & -0.03 \\
\cline { 2 - 6 } & ADC(3) & 4.90 & 1.15 & 0.06 & -0.02 \\
\cline { 2 - 6 } & B3LYP & 4.33 & 1.28 & 0.21 & -0.16 \\
\cline { 2 - 6 } & CAM-B3LYP & 4.40 & 1.27 & 0.21 & -0.16 \\
\hline
\end{tabular}

Table S2: Exciton properties for $S 1$ - S4 states of embelin for ADC2, ADC3 and DFT Xc-functionals. 


\section{Influence of solvation on excitation energies}

\begin{tabular}{lllll}
\hline & $\begin{array}{l}\text { PCM-TDDFT } \\
\text { (CAM-B3LYP) }\end{array}$ & & Expt. \\
\hline & Acetonitrile & DMSO & Methanol & \\
\hline $\mathrm{S}_{1}$ & 3.00 & 2.98 & 2.97 & 2.7552 \\
\hline $\mathrm{S}_{2}$ & 3.67 & 3.66 & 3.65 & \\
\hline $\mathrm{S}_{3}$ & 4.00 & 4.00 & 4.00 & \\
\hline $\mathrm{S}_{4}$ & 4.29 & 4.76 & 4.48 & \\
\hline
\end{tabular}

Table S3: Excitation energies of embelin in different solvents (oscillator strength in brackets) not taking explicit influence of solvation into account.

\section{Embelin as proton donor or acceptor}

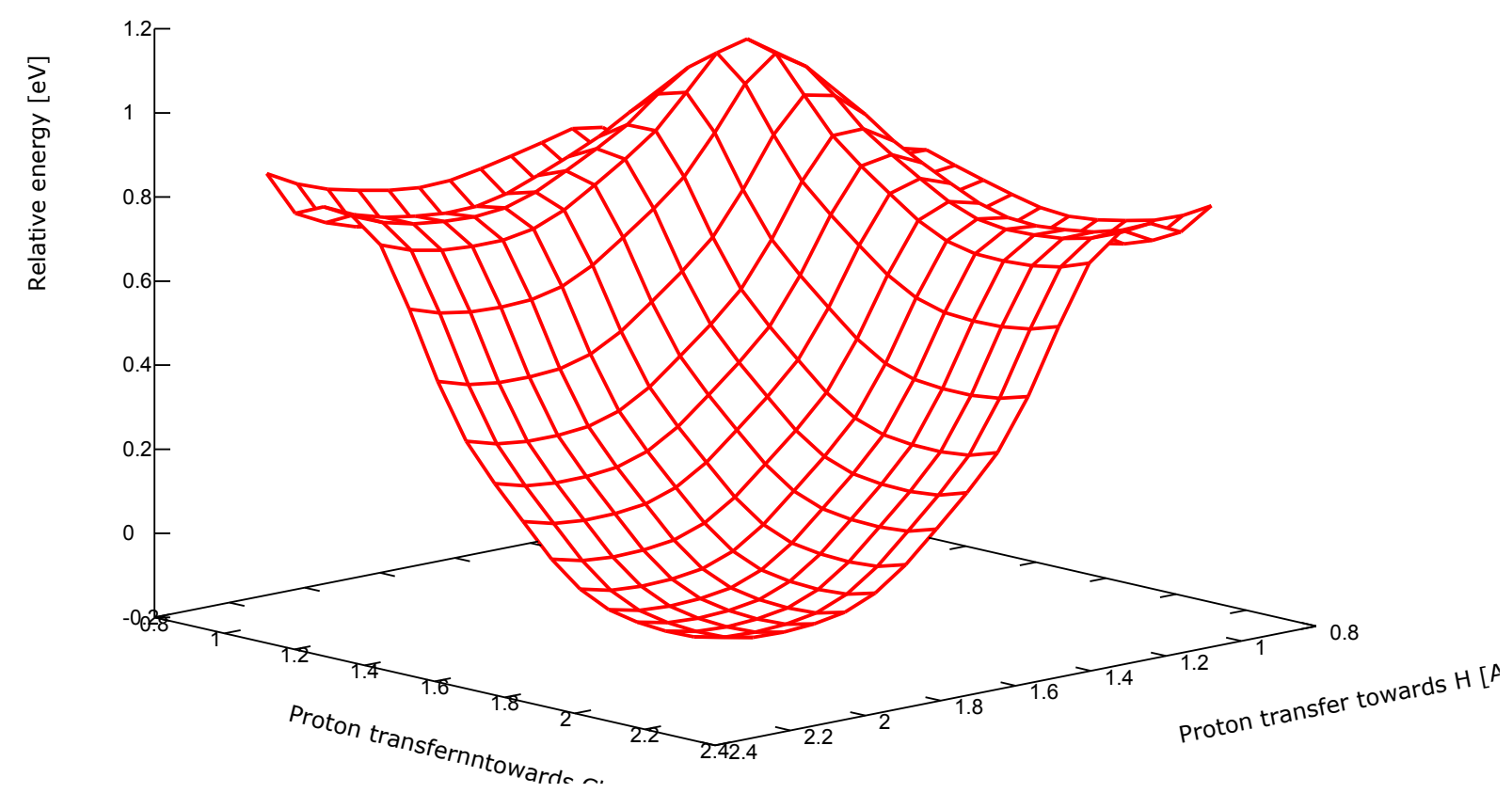

Figure S3: Corresponding Relaxed ground state PES for embelin as a proton donor. The graph was generated by simultaneously moving protons from the hydroxy groups towards the keto groups as shown in Figure S1 above. 


\begin{tabular}{|l|c|c|c|c|}
\hline Parameter & $\mathbf{I}_{\mathbf{H}}(\mathbf{g s})$ & $\mathbf{I}_{\mathrm{CH}}(\mathbf{g s})$ & $\mathbf{I}_{\mathrm{H}}(\mathbf{e s})$ & $\mathbf{I}_{\mathrm{CH}_{3}}(\mathbf{e s})$ \\
\hline O3-C9 & 1.32 & 1.28 & 1.33 & 1.23 \\
\hline C9-C11 & 1.37 & 1.38 & 1.38 & 1.45 \\
\hline C10-C11 & 1.38 & 1.40 & 1.39 & 1.45 \\
\hline C10-O4 & 1.31 & 1.27 & 1.34 & 1.23 \\
\hline O2-C8 & 1.26 & 1.31 & 1.23 & 1.34 \\
\hline C8-C6 & 1.4 & 1.39 & 1.46 & 1.40 \\
\hline C6-C7 & 1.39 & 1.38 & 1.5 & 1.40 \\
\hline C7-01 & 1.27 & 1.39 & 1.23 & 1.34 \\
\hline C8-C6-C7 (degrees) & 114.32 & 112.94 & 120.06 & 118.34 \\
\hline C9-C11-C10 (degrees) & 117.95 & 119.10 & 120.14 & 121.46 \\
\hline
\end{tabular}

Table S4: Geometry differences between intermediate structures formed on the ground and excited state PES of embelin. It is evident that at the excited state geometry, the dihedral angles increase in correspondence with the coupling vectors. See Figure 9 in text

\section{NHO and NBO orbital representation}

FILE. 35

Model 1.1 MO $32 / 414$ NHO $03($ C9)
Occupancy $=2.0$

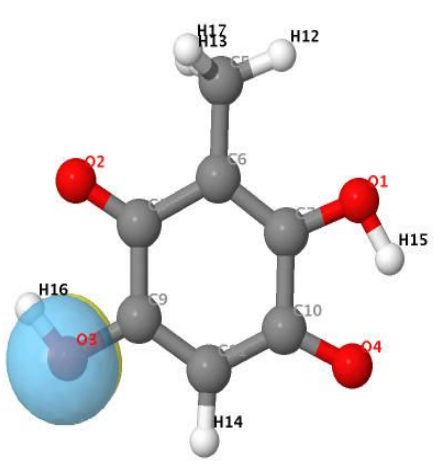

FILE. 35
Model 1.1 MO $33 / 414$ NHO C9(03)

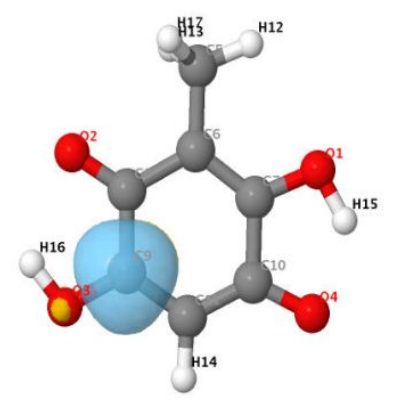

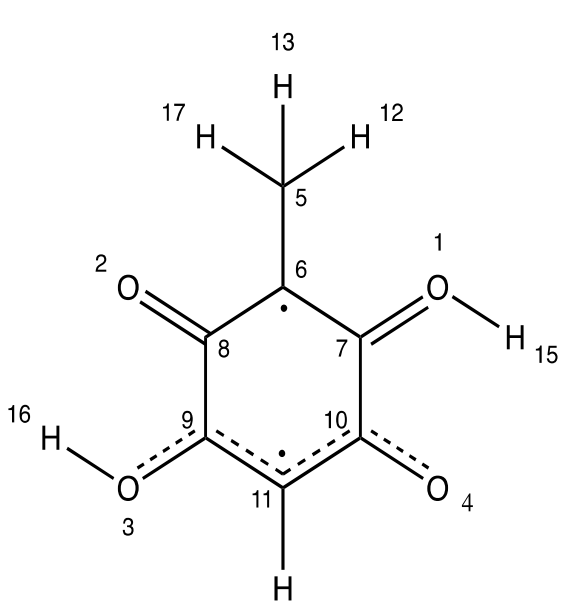

FILE. 35
Model 1.1 MO $38 / 414$ NHO 04 (C10)

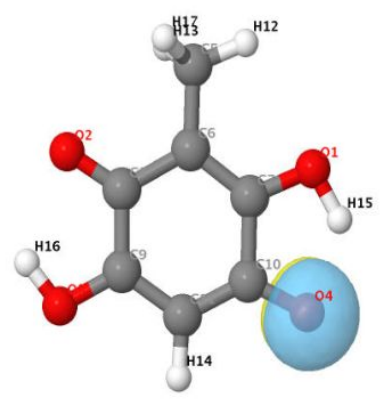

FILE. 35
Model 1.1 MO $39 / 414$ NHO C10(04)

FILE. 35
Modei 1.1 MO $19 / 414$ NHO C11(1p)

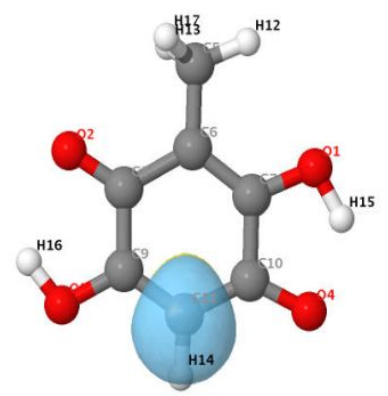

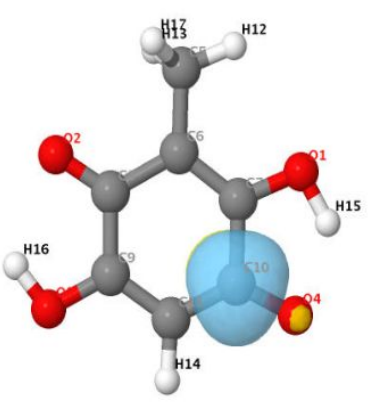



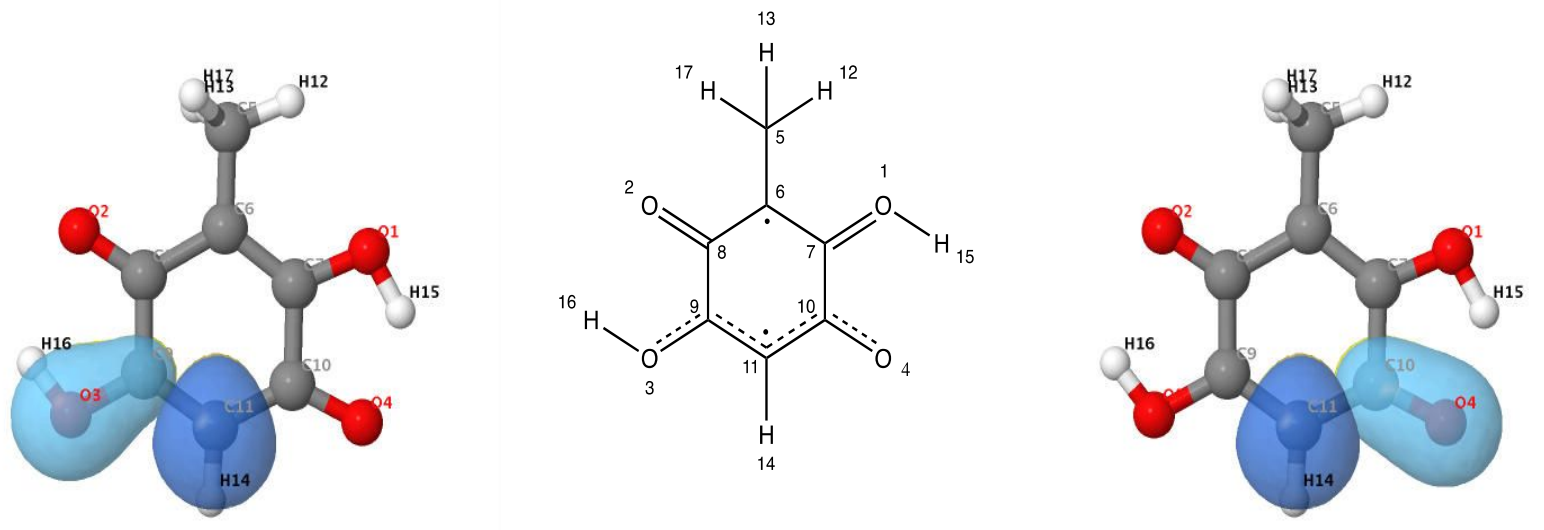

Figure S5: S1 excited states NBO hyperbond orbitals depicting electron from 03 to 04 . This electron delocalization effect is responsible for the stability of embelin at the excited state yielding $I_{H}$ Similar behaviour is not observed for $I_{C H 3}$ 